KYUSHU-HET-130

\title{
Grand Gauge-Higgs Unification
}

\author{
Kentaro Kojima ${ }^{1}$ Kazunori Takenaga ${ }^{2}$ and Toshifumi Yamashita ${ }^{3}$ \\ ${ }^{1}$ Center for Research and Advancement in Higher Education, \\ Kyushu University, Fukuoka 819-0395, Japan \\ ${ }^{2}$ Faculty of Health Science, Kumamoto Health Science University, \\ Izumi-machi, Kumamoto 861-5598, Japan and \\ ${ }^{3}$ Department of Physics, Nagoya University, Nagoya 464-8602, Japan
}

\begin{abstract}
We propose a novel way to break grand unified gauge symmetries via the Hosotani mechanism in models that can accommodate chiral fermions. Adjoint scalar fields are realized through the so-called diagonal embedding method which is often used in the heterotic string theory. We calculate the one-loop effective potential of the adjoint scalar field in a five dimensional model compactified on an $S^{1} / \mathbb{Z}_{2}$ orbifold, as an illustration. It turns out that the potential is basically the same as the one in an $S^{1}$ model, and thus the results in literatures, in addition to the chiral fermions, can be realized easily.
\end{abstract}

PACS numbers: 11.10.Kk, 11.15.Ex, 12.10.Dm

\section{INTRODUCTION}

The Hosotani mechanism [1] is one of the most interesting features possessed by models with topological extra dimensions, and has been studied extensively in the literature [2 4]. In this mechanism, zero-modes of the extra-dimensional components of the higher dimensional gauge fields acquire non-vanishing vacuum expectation values (VEVs) to break the gauge symmetry. Since the extra dimensional components behave as adjoint scalar fields at low energy [2, 3], the mechanism has mainly been applied to the grand unified theories (GUTs) in the last century. The idea, however, encounters the difficulty that one cannot obtain the chiral fermions, and thus it is not phenomenologically viable.

After the work in Ref. [5] on the orbifold symmetry breaking within the field theoretical framework, which opens a way to realize scalar fields in the fundamental representation from the extra-dimensional components of the higher dimensional gauge fields, the Hosotani mechanism is often applied to the electroweak symmetry breaking. In this scenario, one can obtain the chiral fermions, and in addition, it gives a new approach to the hierarchy problem [3] without using supersymmetry. The scenario is called gauge-Higgs unification and has been studied from various points of view [4].

It is natural to ask whether or not one can apply the Hosotani mechanism to the GUTs without contradicting with the existence of the chiral fermion. Our aim in this letter is to establish a way to realize this application in the case of the orbifold compactification. This is very attractive because the gauge symmetry-breaking patterns are completely determined by the calculable dynamics independently of details of the unknown ultraviolet comple-

\footnotetext{
*Electronic address: kojima@rche.kyushu-u.ac.jp

${ }^{\dagger}$ Electronic address: takenaga@kumamoto-hsu.ac.jp

${ }^{\ddagger}$ Electronic address: yamasita@eken.phys.nagoya-u.ac.jp
}

tion, thanks to the finiteness of the Higgs effective potential [3]. This is in great contrast to the orbifold breaking in which the symmetry-breaking pattern is freely chosen by boundary conditions (BCs). In addition, at the same time, one can incorporate the chiral fermions easily.

There is, however, a difficulty in this attempt. The massless adjoint scalar fields, which originate from the extra-dimensional components, tend to be projected out at low energy in models that realize the chiral fermions. This is caused by the difference between the BCs of the four-dimensional (4D) vector components and those of the extra-dimensional ones. It is actually possible that they have a common BC, when there are directions compactified on a certain manifold, such as a torus. The chiral fermions, however, disappear when such directions exist.

This difficulty is shared with the heterotic string theory [6]. Since this theory is expected to contain the standard model, it has been thoroughly studied and a way to overcome the difficulty was proposed, through the socalled diagonal embedding method [7-9]. This method extracts the diagonal part of $n$-copies of a gauge symmetry $G,(G)^{n}$, by, for instance, a $\mathbb{Z}_{n}$ orbifold action that permutes them. In view of the orbifold projection, the eigenvalues of the permutation are given as $e^{2 \pi i k / n}$ $(k=0,1, \cdots, n-1)$. In particular, the gauge factor with $k=0$ corresponds to the diagonal part and remain unbroken by the orbifold action. In other words, the remaining gauge symmetry in the $4 \mathrm{D}$ effective theory is embedded into the diagonal part. A point is that if the phases of other eigenstates cancel those of the extra-dimensional components of the gauge field which comes from the geometrical twist, adjoint scalars appear in the massless spectrum [9].

We find no reasons that forbid the application of the same method to our phenomenological setup and we examine this possibility. An advantage of working in a simple field theoretical setup is that it is much easier to calculate the quantum corrections that tell us the positions of vacua. By this, it is possible to determine the gauge 
symmetry-breaking patterns dynamically. In this letter, as an illustration, we work on $S^{1} / \mathbb{Z}_{2}$ models since extension to models with a more complex orbifold such as $T^{2} / \mathbb{Z}_{3}$ is straightforward.

\section{DIAGONAL EMBEDDING IN $S^{1} / \mathbb{Z}_{2}$ MODEL}

Let us rephrase the diagonal embedding method in terms of the BCs. In the $S^{1} / \mathbb{Z}_{2}$ orbifold model, we should fix the $\mathrm{BCs}$ with respect to the reflections of the 5thdimensional coordinate, $y$, at the two fixed points, $y_{0}=0$ and $y_{\pi}=\pi R$. We choose them so that two-copies of the gauge symmetry $G$ are exchanged [15]. For this purpose, the Lagrangian should be symmetric under the exchange. Namely, we consider models with a $G \times G \times \mathbb{Z}_{2}$ symmetry. We formally distinguish the two gauge factors with indices, as $G_{1}$ and $G_{2}$. Their gauge fields and generators are named as $A_{M}^{(1)}$ and $A_{M}^{(2)}$, and $T_{1}^{a}$ and $T_{2}^{a}$, respectively, where $M=\mu(=0-3), 5$ is a $5 \mathrm{D}$ Lorentz index.

To be more concrete, we choose the BCs as

$$
A_{\mu}^{(1)}\left(y_{i}-y\right)=A_{\mu}^{(2)}\left(y_{i}+y\right)
$$

which are rewritten as

$$
A_{\mu}^{( \pm)}\left(y_{i}-y\right)= \pm A_{\mu}^{( \pm)}\left(y_{i}+y\right)
$$

where we have defined $X^{( \pm)}=\left(X^{(1)} \pm X^{(2)}\right) / \sqrt{2}$ and used $y_{i}(i=0, \pi)$. Since $A_{5}$ has the parity opposite to $A_{\mu}$ to keep the Lagrangian invariant, they lead to

$$
A_{5}^{( \pm)}\left(y_{i}-y\right)=\mp A_{5}^{( \pm)}\left(y_{i}+y\right) \text {. }
$$

The BCs (2) imply that the gauge symmetries $G_{1} \times G_{2}$ are broken down to their diagonal part $G_{\text {diag }}$ whose gauge field is $A_{\mu}^{(+)}$, while those in (3) show that the zero-mode of the extra-dimensional component $A_{5}^{(-)}$exists, which behaves as the adjoint representation of $G_{\text {diag }}$ as we will see soon. In this way, a massless adjoint scalar is obtained in the orbifold model.

The Wilson line in this case is given as

$$
W=\exp \left(i \int_{0}^{2 \pi R} g\left({A_{5}^{(1)}}^{a} T_{1}^{a}+{A_{5}^{(2)}}^{a} T_{2}^{a}\right) d y\right)=\exp \left(i \int_{0}^{2 \pi R} \frac{g}{\sqrt{2}}\left(A_{5}^{(+)^{a}}\left(T_{1}^{a}+T_{2}^{a}\right)+A_{5}^{(-)^{a}}\left(T_{1}^{a}-T_{2}^{a}\right)\right) d y\right)
$$

where $g$ is the common gauge coupling constant. Taking the commutation relations with the generators of the diagonal group,

$$
\left[T_{1}^{a}+T_{2}^{a}, T_{1}^{b} \pm T_{2}^{b}\right]=i f^{a b c}\left(T_{1}^{c} \pm T_{2}^{c}\right),
$$

we see that both combinations $\left(T_{1} \pm T_{2}\right)$ behave as adjoint representation under the diagonal group. Note that the normalizations of $\left(T_{1} \pm T_{2}\right)$ have no factor of $1 / \sqrt{2}$ to satisfy the commutation relation (5). This results in the suppressed gauge coupling of $G_{\text {diag }}$ compared to the original one, $g_{\text {diag }}=g / \sqrt{2}$.

Since only the combination $A_{5}^{(-)}$has zero-modes, we set the VEVs of $A_{5}$ as

$$
\left\langle\frac{g}{\sqrt{2}} A_{5}^{(-)^{a}}\right\rangle=\left\langle g A_{5}^{(1)^{a}}\right\rangle=-\left\langle g A_{5}^{(2)^{a}}\right\rangle=\frac{\theta^{a}}{\pi R} .
$$

Then the VEV of the Wilson line becomes

$$
\langle W\rangle=\exp \left(i \theta^{a} 2\left(T_{1}^{a}-T_{2}^{a}\right)\right) .
$$

\section{FERMIONS}

Because of the $\mathbb{Z}_{2}$ symmetry which is required to impose the BCs that exchange the two gauge groups, when we introduce a fermion, $\Psi^{(1)}\left(\mathbf{R}_{\mathbf{1}}, \mathbf{R}_{\mathbf{2}}\right)$ with $\mathbf{R}_{\mathbf{1}} \neq \mathbf{R}_{\mathbf{2}}$ where $\mathbf{R}_{\mathbf{1}}$ and $\mathbf{R}_{\mathbf{2}}$ denote its representations of $G_{1}$ and $G_{2}$, respectively, its $\mathbb{Z}_{2}$ partner $\Psi^{(2)}\left(\mathbf{R}_{2}, \mathbf{R}_{1}\right)$ should be also introduced. These fermions behave as a reducible representation $\mathbf{R}_{\mathbf{1}} \times \mathbf{R}_{\mathbf{2}}$ under the residual diagonal gauge groups. To keep the Lagrangian invariant, their BCs are given as

$$
\Psi^{(1)}\left(y_{i}-y\right)=\eta_{\Psi} \gamma^{5} \Psi^{(2)}\left(y_{i}+y\right),
$$

where $\eta_{\Psi}$ is a parameter being $\eta_{\Psi}^{2}=1$. Namely,

$$
\Psi^{( \pm)}\left(y_{i}-y\right)= \pm \eta_{\Psi} \gamma^{5} \Psi^{( \pm)}\left(y_{i}+y\right) .
$$

This implies that a vector-like pair of the fermion zeromodes in the reducible representation $\mathbf{R}_{\mathbf{1}} \times \mathbf{R}_{\mathbf{2}}$ appears.

In the case with $\mathbf{R}_{\mathbf{1}}=\mathbf{R}_{\mathbf{2}}(=\mathbf{R})$, a single fermion $\psi^{\alpha \beta}$ is allowed, where $\alpha$ and $\beta$ are the indices of the representation $\mathbf{R}$ on which the elements of $G_{1}$ and $G_{2}$, respectively, act. It behaves as a reducible representation of the diagonal group:

$$
\mathbf{R} \times \mathbf{R}=\left(\mathbf{R}_{s 1}+\cdots\right)+\left(\mathbf{R}_{a 1}+\cdots\right),
$$

where " $s$ " and " $a$ " denote symmetric and anti-symmetric products, respectively. We define $\psi_{ \pm}^{\alpha \beta}=\left(\psi^{\alpha \beta} \pm \psi^{\beta \alpha}\right) / 2$ so that $\psi_{+}$and $\psi_{-}$consist of $\mathbf{R}_{s i}$ and $\mathbf{R}_{a i}$, respectively. 
Its BCs are

$$
\begin{aligned}
\psi^{\alpha \beta}\left(y_{i}-y\right) & =\eta_{\psi} \gamma^{5} \psi^{\beta \alpha}\left(y_{i}+y\right), \\
\text { or } \quad & \psi_{ \pm}^{\alpha \beta}\left(y_{i}-y\right)= \pm \eta_{\psi} \gamma^{5} \psi_{ \pm}^{\alpha \beta}\left(y_{i}+y\right) .
\end{aligned}
$$

With this, we see that chiral zero-modes appear in this case [16].

The interaction terms among the fermions and the 5D gauge fields are written as

$$
\begin{aligned}
& g\left(\begin{array}{ll}
\bar{\Psi}^{(1)} & \bar{\Psi}^{(2)}
\end{array}\right)\left(\begin{array}{cc}
A^{(1)^{a}} T_{\mathbf{R}_{\mathbf{1}}}^{a}+\not A^{(2)^{a}} T_{\mathbf{R}_{\mathbf{2}}}^{a} & 0 \\
0 & \not^{(1)^{a}} T_{\mathbf{R}_{\mathbf{2}}}^{a}+\not^{(2)^{a}} T_{\mathbf{R}_{\mathbf{1}}}^{a}
\end{array}\right)\left(\begin{array}{l}
\Psi^{(1)} \\
\Psi^{(2)}
\end{array}\right)
\end{aligned}
$$

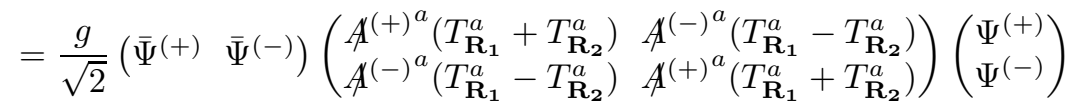

$$
\begin{aligned}
& \ni \bar{\Psi}^{( \pm)} \frac{\theta^{a}}{\pi R}\left(T_{\mathbf{R}_{\mathbf{1}}}^{a}-T_{\mathbf{R}_{\mathbf{2}}}^{a}\right) i \gamma^{5} \Psi^{(\mp)} .
\end{aligned}
$$

Here, $T_{\mathbf{r}}$ is the generator on the representation $\mathbf{r}$ and $A=A_{M} \Gamma^{M}$ with $\Gamma^{M}=\left(\gamma^{\mu}, i \gamma^{5}\right)$. Note that the last two expressions also hold for $\psi$, with the replacements $\Psi^{( \pm)} \rightarrow \psi_{ \pm}$and $T_{\mathbf{R}_{1}}\left(T_{\mathbf{R}_{2}}\right)$ by $T_{\mathbf{R}}$ that act on the first (second) index of $\psi_{ \pm}$. Thus, we concentrate on $\Psi$ in the remaining in this section, except for specially mentioned comments.

On the background (6), the mass terms for the $n$-th Kaluza-Klein $(\mathrm{KK})$ modes of $\Psi^{( \pm)}$, with $n$ being a nonnegative integer, are

$$
\mathcal{L}_{K K}=\frac{-1}{R}\left(\begin{array}{ll}
\bar{\Psi}_{n}^{(+)} & \bar{\Psi}_{n}^{(-)}
\end{array}\right)_{\chi^{\prime}}\left(\begin{array}{cc}
-i \theta^{a}\left(T_{\mathbf{R}_{1}}^{a}-T_{\mathbf{R}_{2}}^{a}\right) / \pi & -i \theta^{a}\left(T_{\mathbf{R}_{1}}^{a}-T_{\mathbf{R}_{2}}^{a}\right) / \pi \\
n
\end{array}\right) \gamma_{5}\left(\begin{array}{l}
\Psi_{n}^{(+)} \\
\Psi_{n}^{(-)}
\end{array}\right)_{\chi}+c . c .
$$

where $\chi$ and $\chi^{\prime}(\neq \chi)$ denote the chiralities which we fix so that $\left(\Psi^{(+)}\right)_{\chi}$ becomes even. Diagonalizing the above matrix and rearranging phases by the chiral rotation, we find the KK spectrum is given as

$$
m_{K K}^{(n)} R=n+\theta^{a}\left(T_{\mathbf{R}_{1}}^{a}-T_{\mathbf{R}_{\mathbf{2}}}^{a}\right) / \pi, \quad n \in \mathbb{Z} .
$$

Let us note that the KK spectrum is basically the same form as the one obtained for the $S^{1}$ compactification, though the effect of $\theta^{a}$ is non-trivial as discussed soon. With this expression, it is easy to calculate the contributions to the one-loop Higgs effective potential [1, 2].

It is interesting to see that for the fermions with $\mathbf{R}_{\mathbf{2}}$ being trivial, the situation is completely the same as the $S^{1}$ compactification with the same radius $R$ and the original gauge coupling constant $g$. Note that the $4 \mathrm{D}$ effective gauge couplings are the same as that of the remaining $G_{\text {diag }}: g_{\text {diag }}^{2} / L_{S^{1} / \mathbb{Z}_{2}}=g_{S^{1}}^{2} / L_{S^{1}}=g^{2} /(2 \pi R)$. This is understood by latticizing the extra-dimension [11] as shown in FIG. 1. From this figure, we see the resulting Moose diagram looks the same as the one for the $S^{1}$ model. This means that what can be done in the $S^{1}$ models in the literatures are easily reproduced, while the chiral fermions can be put on the branes.

For the fermions with non-trivial $\mathbf{R}_{\mathbf{1}}$ and $\mathbf{R}_{\mathbf{2}}$, the mass spectra (and thus the contributions to the one-loop Higgs effective potential) are the same as the one of the fermion in the $\mathbf{R}_{\mathbf{1}} \times \mathbf{R}_{\mathbf{2}}{ }^{*}$ (reducible) representation in the $S^{1}$ model, while they behave as $\mathbf{R}_{\mathbf{1}} \times \mathbf{R}_{\mathbf{2}}$ representations under the remaining gauge symmetry $G_{D}$. This gives a difference between our model and the $S^{1}$ model, which is again understood via the latticized picture as these fermions seem to have "non-local" interactions with the gauge bosons in two distant sites in view of the $S^{1}$ side.

Special comments on $\psi$ are in order. As mentioned before, the above expressions are also for the case of $\psi$. Since the representation of $\psi_{+}$is, however, different from that of $\psi_{-}$in this case, it is clear that not all of the zeromodes acquire masses. Instead, some components that are vector-like with respect to the unbroken subgroup

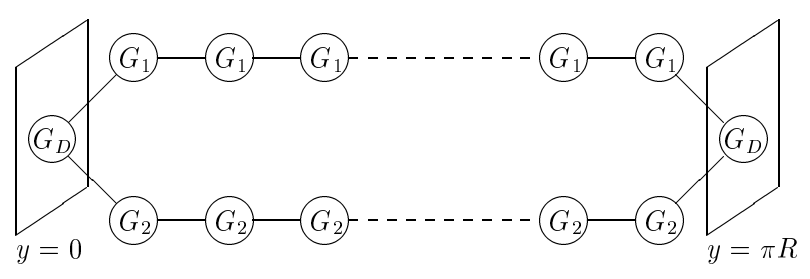

FIG. 1: The latticized picture of $G_{1} \times G_{2} \rightarrow G_{D}$ model on $S^{1} / \mathbb{Z}_{2}$. 
against the VEV of $A_{5}$ become massive. The other components are the eigenstates of $\theta^{a}\left(T_{\mathbf{R}_{\mathbf{1}}}^{a}-T_{\mathbf{R}_{\mathbf{2}}}^{a}\right)$ with vanishing eigenvalues and thus remain massless. These modes can be chiral.

Let us consider an example of an $S U(5)$ model with $\psi(\mathbf{5})$ where the VEV of $A_{5}$ breaks $S U(5)$ into $S U(3)_{C} \times$ $S U(2)_{L} \times U(1)_{Y}$. This $\psi$ is divided into $\psi_{+}(\mathbf{1 5})$ and $\psi_{-}(\mathbf{1 0})$, which are decomposed as

$$
\begin{aligned}
& \mathbf{1 5} \rightarrow(\mathbf{3}, \mathbf{2})_{1 / 6}+(\mathbf{6}, \mathbf{1})_{-2 / 3}+(\mathbf{1}, \mathbf{3})_{1}, \\
& \mathbf{1 0} \rightarrow(\mathbf{3}, \mathbf{2})_{1 / 6}+(\overline{\mathbf{3}}, \mathbf{1})_{-2 / 3}+(\mathbf{1}, \mathbf{1})_{1} .
\end{aligned}
$$

The component $(\mathbf{3}, \mathbf{2})_{1 / 6}$ becomes massive due to the $\mathrm{VEV}$, while the others remain massless. It is easy to see the latter are the eigenstates of $\theta^{a}\left(T_{\mathbf{R}_{1}}^{a}-T_{\mathbf{R}_{2}}^{a}\right)$ with zero eigenvalues in this case.

\section{APPLICATIONS}

In this section, we shall discuss applications to $S U(5)$ models. The literature [1, 2] indicates that it is not easy to realize vacua where the $S U(5)$ symmetry is broken to $S U(3) \times S U(2) \times U(1)$ as the global minimum of the potential in the $S^{1}$ framework. Since the potential in our setup is basically the same as those in the $S^{1}$ models, unfortunately, the same conclusion would be applied to our case.

It is, however, not so important whether we reside on the global minimum or on a local minimum, as far as the lifetime of the local minimum is much longer than the age of universe. In this view, the analysis in Ref. [12] is noteworthy. There, it is claimed that the desired vacuum is realized as a local minimum when one fermion in $\mathbf{5}$ and 10, respectively, one scalar in $\mathbf{5}$ and three scalars in $\mathbf{1 5}$ are introduced. Though we find that the precise point considered in Ref. [12] is not even extremal, there is another point, $\theta^{a} T^{a} / \pi=\operatorname{diag}(2,2,2,-3,-3) / 2$, being actually a local minimum where the desirable symmetry breaking occurs. Since it is not easy to keep the scalars light against the quantum corrections, we would like to replace them by anti-periodic fermions which have a similar effect as the periodic scalars [13]. It is not difficult to check the point remains a local minimum after this modification.

In this way, we can utilize the results in the literature that investigate $S^{1}$ models while the chiral fermions can be put on the branes in our setup. We hope that this letter revives the researches in the literature that have been abandoned because of the lack of the chiral fermions. We leave concrete model building as future works [17].

\section{Acknowledgments}

K.T. is supported in part by a Grant-in-Aid for Science Research (No. 21540285) from the Japanese Ministry of Education, Science, Sports and Culture. The work of T.Y. was partially supported by the Japanese Society for the Promotion of Science.
[1] Y. Hosotani, Phys. Lett. B 126, 309 (1983); Ann. of Phys. 190, 233 (1989); Phys. Lett. B 129, 193 (1983); Phys. Rev. D 29, 731 (1984).

[2] A. Higuchi and L. Parker, Phys. Rev. D 37, 2853 (1988); A. T. Davies and A. McLachlan, Phys. Lett. B 200, 305 (1988); Nucl. Phys. B 317, 237 (1989); J. E. Hetrick and C. L. Ho, Phys. Rev. D 40, 4085 (1989); A. McLachlan, Nucl. Phys. B 338, 188 (1990); C. L. Ho and Y. Hosotani, Nucl. Phys. B 345, 445 (1990); H. Hatanaka, Prog. Theor. Phys. 102, 407 (1999); K. Takenaga, Phys. Lett. B 570, 244 (2003); N. Haba, K. Takenaga and T. Yamashita, Phys. Lett. B 605, 355 (2005).

[3] N. V. Krasnikov, Phys. Lett. B 273, 246 (1991); H. Hatanaka, T. Inami and C. S. Lim, Mod. Phys. Lett. A 13, 2601 (1998); G. R. Dvali, S. Randjbar-Daemi and R. Tabbash, Phys. Rev. D 65, 064021 (2002); N. ArkaniHamed, A. G. Cohen and H. Georgi, Phys. Lett. B 513, 232 (2001); I. Antoniadis, K. Benakli and M. Quiros, New J. Phys. 3, 20 (2001); N. Maru and T. Yamashita, Nucl. Phys. B 754, 127 (2006); Y. Hosotani, N. Maru, K. Takenaga and T. Yamashita, Prog. Theor. Phys. 118, 1053 (2007).

[4] C. Csaki, C. Grojean and H. Murayama, Phys. Rev. D 67, 085012 (2003); G. Burdman and Y. Nomura, Nucl. Phys. B 656, 3 (2003); N. Haba, M. Harada, Y. Hosotani and Y. Kawamura, Nucl. Phys. B 657, 169
(2003) [Erratum-ibid. B 669, 381 (2003)]; N. Haba and Y. Shimizu, Phys. Rev. D 67, 095001 (2003) [Errata; 69, 059902 (2004)]; I. Gogoladze, Y. Mimura and S. Nandi, Phys. Lett. B 560, 204 (2003); C. A. Scrucca, M. Serone and L. Silvestrini, Nucl. Phys. B 669, 128 (2003); R. Contino, Y. Nomura and A. Pomarol, Nucl. Phys. B 671, 148 (2003); K. Agashe, R. Contino and A. Pomarol, Nucl. Phys. B 719, 165 (2005); K. Agashe and R. Contino, Nucl. Phys. B 742, 59 (2006); A. D. Medina, N. R. Shah and C. E. M. Wagner, Phys. Rev. D 76, 095010 (2007); Y. Hosotani and Y. Sakamura, Prog. Theor. Phys. 118, 935 (2007); Y. Hosotani, P. Ko and M. Tanaka, Phys. Lett. B 680, 179 (2009); C. S. Lim and N. Maru, Phys. Lett. B 653, 320 (2007); N. Haba, Y. Sakamura and T. Yamashita, JHEP 0907, 020 (2009); JHEP 1003, 069 (2010).

[5] Y. Kawamura, Prog. Theor. Phys. 103, 613 (2000); ibid 105, 691 (2001); ibid 105, 999 (2001).

[6] D. J. Gross, J. A. Harvey, E. J. Martinec and R. Rohm, Phys. Rev. Lett. 54, 502 (1985); Nucl. Phys. B 256, 253 (1985); Nucl. Phys. B 267, 75 (1986).

[7] K. R. Dienes and J. March-Russell, Nucl. Phys. B 479, 113 (1996).

[8] D. C. Lewellen, Nucl. Phys. B 337, 61 (1990); G. Aldazabal, A. Font, L. E. Ibanez and A. M. Uranga, Nucl. Phys. B 452, 3 (1995); J. Erler, Nucl. Phys. B 475, 597 
(1996); Z. Kakushadze and S. H. H. Tye, Phys. Rev. D 55, 7878 (1997); Phys. Rev. D 55, 7896 (1997).

[9] M. Ito et al., Phys. Rev. D 83, 091703 (2011).

[10] M. Regis, M. Serone and P. Ullio, JHEP 0703, 084 (2007); G. Panico, E. Ponton, J. Santiago and M. Serone, Phys. Rev. D 77, 115012 (2008).

[11] N. Arkani-Hamed, A. G. Cohen and H. Georgi, Phys. Rev. Lett. 86, 4757 (2001).

[12] V. B. Svetovoi and N. G. Khariton, Sov. J. Nucl. Phys. 43 (2), 280 (1986); Yad. Fiz. 43 (1986) 438.

[13] N. Haba, Y. Hosotani, Y. Kawamura and T. Yamashita, Phys. Rev. D 70, 015010 (2004); N. Haba, K. Takenaga and T. Yamashita, Phys. Lett. B 615, 247 (2005); N. Haba, S. Matsumoto, N. Okada and T. Yamashita,
JHEP 0602, 073 (2006).

[14] T. Yamashita, arXiv:1106.3229 [hep-ph].

[15] A similar BC is adopted in Ref. [10] to improve the naturalness and realize a dark matter candidate, at one of the fixed points.

[16] In addition, as usual, chiral fermions can be put on each brane at $y=y_{i}$

[17] In Ref. [14], for instance, it is claimed that the so-called doublet-triplet splitting problem can be solved in supersymmetric version of this scenario where light adjoint chiral multiplets are predicted. They have masses of the supersymmetry-breaking scale and thus are testable at the $\mathrm{TeV}$-scale collider experiments. 